\title{
Spinal Manipulative Therapy for Acute Low Back Pain
}

\author{
PHIL S. SIZER, JR, PT, PhD
}

would like to commend Hancock and colleagues on their article entitled 'Spinal Manipulative Therapy for Acute Low Back Pain; A Clinical Perspective.' My interest in this paper was triggered by their willingness to discuss questions that practitioners in the manual therapy community may be less comfortable with, based on their own commitment to a particular approach or model used in examination and care. The authors did not so much offer a host of definitive answers, but rather provided suggestions in the wake of many unanswered questions. For this they should be thanked, because it could stimulate a healthy surge towards further evaluating the important questions daily confronted by spinal manipulative therapy (SMT) providers.

While SMT is an accepted component in the management of acute LBP, one of the greatest challenges is the timing of when a clinician should implement SMT in the management plan. The authors suggested that, prior to SMT implementation, screening should be engaged in order to rule out fracture or serious pathology. Screening is clearly needed as a preliminary component of managing spine pain ${ }^{1}$. However, greater attention should be given to this practice than is presently exercised, considering the low rate of routine examination for red flags in primary care practice settings ${ }^{2}$ using guideline-recommended approaches ${ }^{3,4}$.
The presence of serious examination findings such as pulsatile abdominal masses, unexplained neurological deficits, and recent bowel and bladder changes, suggests serious pathology outside the domain of musculoskeletal disorders and may require immediate intervention by an appropriate specialist ${ }^{1,5}$. Other findings that include a cancer history, long-term corticosteroid use, metabolic bone disorder history, age greater than 50 years, unexplained weight loss, and failure of conservative management require further patient questioning, selected examination methods, and the evaluation of the historical and examination findings in clusters. For example, a history of long-term corticosteroid use and an age greater than 50 each do not warrant immediate attention by a specialist. However, when clustered, the likelihood of a spinal compression fracture is dramatically increased, thus suggesting a specialist's attention ${ }^{6}$.

The authors' concern over fractures and cancer is well received. However, besides fracture and cancer, acute low back pain patients are at risk for other serious pathologies. For example, pyogenic infectious disease can develop in this region resulting in fever, malaise, potential bowel and bladder disturbances and severe low back pain. Vertebral osteomyelitis and pyogenic spondylodiscitis can develop after streptococcal endocarditis, direct inoculation, discography, nucleoplasty, colonoscopy, biopsy, or oocyte retrieval for in-vitro fertilization ${ }^{5,-12}$.

Spontaneous pyogenic infection can form in the lumbar zygapophyseal joints as well, especially in patients with a history of drug abuse or previous spinal instrumentation $^{13}$. Serious arthritides can occur in both the zygapophyseal joints and sacroiliac joints, thus meriting a screening process. Rheumatoid arthritis, systemic lupus erythematous, ankylosing spondylitis, brucellosis and Reiter's syndrome can all produce the same host of symptoms suggesting the need for screening ${ }^{14-17}$.

I was intrigued when the authors suggested that studies evaluating the efficacy of SMT have produced small and or contradictory results, and when they reported plausible reasons for these differences. It is apparent that these authors consider the present model of $1^{\text {st }}$ line care that uses advice and appropriately dosed Paracetamol (acetaminophen or Tylenol) as the best choice for the patient. The investigators' argument regarding the limits to studies involving clinical prediction rules (CPR)-based decisions are reasonable and recommending the global use of CPR as the standard for care should be limited until CPR studies are sufficiently evaluated in a valid fashion for quality. However, putting this limit to CPR-based decisions aside, one is impelled to ask why SMT need be considered $2^{\text {nd }}$ line care. The authors should be cautioned

Professor \& Program Director, ScD Program in Physical Therapy, Director, Clinical Musculoskeletal Research Laboratory, Department of Rehabilitation Sciences, School of Allied Health Sciences, Texas Tech University Health Sciences Center, Lubbock, TX, USA 
that instead of being considered $2^{\text {nd }}$ line care, SMT should be considered a nonpharmaceutical $1^{\text {st }}$ line care approach. While the side effects for Paracetamol are small, they still exist and one could argue that SMT may serve as a healthy alternative to the contemporary primary care practice model.

Finally, I was gravitated to the authors' reflections on a very meaningful question regarding the value of differences in SMT treatment approaches to the outcome of the care. While the Kent et al systematic review suggested that clinician choice of treatment may not influence treatment effects, those authors admitted that the evidence was limited and a cautionary interpretation is merited ${ }^{18}$. Hancock and colleagues go on to provide a reasonable argument for the accurate selection of the most painful lumbar spinal segment for treatment, the value of segment-specific treatment accompanied by systematic reassessment and treatment modification, and the need for further research investigating the relationship between segmental hypomobility and low back pain and or the effect of SMT. Furthermore, their discussion regarding a lack of evidence supporting the superiority of low- versus high-velocity manipulative therapy inspires the need for further investigations. Yet, biopsychosocial considerations should be included when evaluating the influence of SMT treatment approach selection on treatment outcomes. One must question the role of a patient's confidence in the clinician's treatment selection, as well as the role of the clinician's belief in that selection on the outcome. While these considerations have not been evaluated in terms of their specific influence on the efficacy of SMT for patients with acute low back pain, selected studies suggest a need for this consideration. First, Bialosky et al found that normal subjects with negative expectations for spinal manipulation-induced hypoalgesia experienced a significant increase in pain perception following SMT, suggesting the role of recipient expectation on SMT effects ${ }^{19}$. Second, Daykin and Richardson re- ported that clinicians' pain beliefs, albeit in regards to chronic pain, were determined by their beliefs regarding development of craft knowledge, beliefs about "good" versus "difficult" to-treat patients, and pain beliefs within the therapeutic encounter ${ }^{20}$. While both studies were not centered on the effects of either patient expectations or clinician beliefs in the clinician's encounter with an acute low back pain sufferer, one must consider the role of these factors in the efficacy of any particular treatment approach when a patient and clinician enter the healing relationship. Clearly, more research evaluating these factors is merited with this population.

In summary, I want to thank Hancock and colleagues for bringing these and other issues to light and look forward to the studies that will follow.

\section{REFERENCES}

1. Staal JB, Hlobil H, van Tulder MW, et al. Occupational health guidelines for the management of low back pain: An international comparison. Occup Environ Med 2003;60: 618-626.

2. Bishop PB, Wing PC. Knowledge transfer in family physicians managing patients with acute low back pain: A prospective randomized control trial. Spine J 2006;6:282-288.

3. Bishop PB, Wing PC. Compliance with clinical practice guidelines in family physicians managing worker's compensation board patients with acute lower back pain. Spine J 2003;3:442-450.

4. Gonzalez-Urzelai V, Palacio-Elua L, Lopezde-Munain J. Routine primary care management of acute low back pain: Adherence to clinical guidelines. Eur Spine J 2003;12:589594.

5. Sizer P, Brismée JM, Cook C. Medical screening for red flags in the diagnosis and management of musculoskeletal spine pain. Pain Prac 2007;7:53-71.

6. Jarvik JG, Deyo RA. Diagnostic evaluation of low back pain with emphasis on imaging. Ann Intern Med 2002;137:586-597.

7. Poyanli A, Poyanli O, Akan K, Sencer S. Pneumococcal vertebral osteomyelitis: A unique case with atypical clinical course. Spine 2001;26:2397-2399.
8. Tay BK, Deckey J, Hu SS. Spinal infections. J Am Acad Orthop Surg 2002;10:188-197.

9. Chen F, Lu G, Kang Y, et al. Mucormycosis spondylodiscitis after lumbar disc puncture. Eur Spine J 2006;15:370-376.

10. Lechiche C, Le Moing V, Marchandin H, Chanques G, Atoui N, Reynes J. Spondylodiscitis due to Bacteroides fragilis: Two cases and review. Scand J Infect Dis 2006;38:229231.

11. Debusscher F, Troussel S, van Innis F, Holemans X. Spondylodiscitis after transvaginal oocyte retrieval for in vitro fertilisation. Acta Orthop Belg 2005;71:249-251.

12. Yavasoglu I, Kadikoylu G, Bolaman Z, Senturk T. Spondylodiscitis and Streptoccus viridians endocarditis. I Natl Med Assoc 2005;97:1722-1724.

13. Narvaez J, Nolla JM, Narvaez JA, et al. Spontaneous pyogenic facet joint infection. Semin Arthritis Rheum 2006;35:272-283.

14. Brophy S, Calin A. Definition of disease flare in ankylosing spondylitis: The patients' perspective. J Rheumatol 2002;29:954-958.

15. Yen PS, Lin JF, Chen SY, Lin SZ. Tophaceous gout of the lumbar spine mimicking infectious spondylodiscitis and epidural abscess: MR imaging findings. J Clin Neurosci 2005;12:44-46.

16. Pourbagher A, Pourbagher MA, Savas L, et al. Epidemiologic, clinical, and imaging findings in brucellosis patients with osteoarticular involvement. AJR Am J Roentgenol 2006;187:873-880.

17. Kiss S, Letko E, Qamruddin S, Baltatzis S, Foster CS. Long-term progression, prognosis, and treatment of patients with recurrent ocular manifestations of Reiter's syndrome. Ophthalmology 2003;110:1764-1769.

18. Kent P, Marks D, Pearson W, Keating J. Does clinician treatment choice improve the outcomes of manual therapy for nonspecific low back pain? A meta-analysis. J Manipulative Physiol Ther 2005;28:312-322.

19. Bialosky JE, Bishop MD, Robinson ME, Barabas JA, George SZ. The influence of expectation on spinal manipulation induced hypoalgesia: An experimental study in normal subjects. BMC Musculoskelet Disord 2008;9:19.

20. Daykin AR, Richardson B. Physiotherapists' pain beliefs and their influence on the management of patients with chronic low back pain. Spine 2004;29:783-795. 